\title{
Prognostic factors in older patients with wild-type epidermal growth factor receptor advanced non-small cell lung cancer: a multicenter retrospective study
}

\author{
Yurie Seto $^{1}$, Yoshiko Kaneko ${ }^{1}$, Takako Mouri ${ }^{1}$, Hiroyuki Fujii ${ }^{2}$, Satomi Tanaka ${ }^{1}$, Shinsuke Shiotsu ${ }^{2}$, \\ Osamu Hiranuma ${ }^{3}$, Yoshie Morimoto ${ }^{1}$, Masahiro Iwasaku', Tadaaki Yamada ${ }^{1}$, Junji Uchino ${ }^{1}$, \\ Koichi Takayama ${ }^{1}$ \\ ${ }^{1}$ Department of Pulmonary Medicine, Kyoto Prefectural University of Medicine, Kyoto, Japan; ${ }^{2}$ Department of Respiratory Medicine, Japanese Red \\ Cross Kyoto Daiichi Hospital, Kyoto, Japan; ${ }^{3}$ Department of Respiratory Medicine, Otsu City Hospital, Shiga, Japan \\ Contributions: (I) Conception and design: Y Seto, Y Kaneko; (II) Administrative support: Y Kaneko, K Takayama; (III) Provision of study materials or \\ patients: Y Kaneko, Y Morimoto, M Iwasaku, T Yamada, J Uchino, K Takayama; (IV) Collection and assembly of data: Y Seto; (V) Data analysis and \\ interpretation: Y Seto; (VI) Manuscript writing: All authors; (VII) Final approval of manuscript: All authors. \\ Correspondence to: Yoshiko Kaneko. Department of Pulmonary Medicine, Kyoto Prefectural University of Medicine, Kyoto 602-8566, Japan. \\ Email: kaneko-y@koto.kpu-m.ac.jp.
}

Backgroundk Over $40 \%$ Japanese patients with lung cancer are above 75 years of age. A specific strategy to treat such older patients is necessary because most trials exclude older patients with poor physical health. Herein, we aimed to identify predictive factors associated with overall survival (OS) in older patients by evaluating patient backgrounds and laboratory data before the start of treatment.

Methods: This multicenter retrospective medical chart review study was conducted at three Japanese institutions and involved patients aged 75 years and above with epidermal growth factor receptor (EGFR) mutation-negative advanced non-small cell lung cancer (NSCLC). Of the patients, 75 had received best supportive care (BSC) and 49 mono-chemotherapy or platinum-doublet chemotherapy, including immune checkpoint inhibitors (ICIs). OS after diagnosis was analyzed using the Kaplan-Meier survival analysis. Cox proportional hazard models, which included age, Eastern Cooperative Oncology Group performance status (ECOG PS), staging, serum albumin levels, and receipt of chemotherapy were analyzed.

Results: Age at diagnosis was not shown to be related to OS in patients receiving BSC. In patients aged 81 years and above, the chemotherapy group tended to have longer survival than did the BSC group, but there was no statistically significant difference in the median OS between the two groups due to the very small number of subjects (n: 30 vs. 12, median: 52 vs. 30 weeks, hazard ratio: 0.512, 95\% confidence interval: $0.232-1.130, \mathrm{P}=0.088$ ). The patients' performance status and albumin levels at lung cancer diagnosis had the highest impact on OS in the BSC group.

Conclusions: Careful consideration should be given to the indications of chemotherapy for patients aged 81 years and above with wild-type EGFR advanced non-small lung cancer.

Keywords: Non-small cell lung cancer (NSCLC); older patient; best supportive care (BSC); chemotherapy; performance status

Submitted Jul 28, 2020. Accepted for publication Nov 18, 2020.

doi: $10.21037 /$ tlcr-20-894

View this article at: http://dx.doi.org/10.21037/tlcr-20-894 


\section{Introduction}

In 2016, lung cancer was the leading cause of cancerrelated mortality and the fifth leading cause of death due to any reason, with the World Health Organization (WHO) reporting 1.7 million deaths worldwide (1).

In an analysis in the USA, it was found that approximately half of all lung cancers are diagnosed in people aged $>70$ years, and approximately $15 \%$ are diagnosed in people aged $>80$ years (2). In contrast, in a study in Japan, over $40 \%$ of patients diagnosed with lung cancer were over 75 years old, and over $30 \%$ were $>80$ years old (3). The aged population shows significant heterogeneity; hence, advanced age should not hinder older adults from accessing the most appropriate treatment. Treatment of non-small cell lung cancer (NSCLC) completely differs between epidermal growth factor receptor (EGFR)-positive and EGFRnegative cases.

The therapy for EGFR-positive advanced lung cancer in patients aged 75 years or older helps prolong overall survival (OS) similar to patients aged under 75 years (4).

In contrast, the $3 \mathrm{rd}$ generation chemotherapy is recommended for elderly patients with advanced lung cancer who are negative for EGFR or not indicated for other molecular targeted therapies $(5,6)$.

However, some clinical trials restrict older patients with poor physical status from participation $(2,7,8)$. Some clinical trials have shown an increase in the incidence of adverse events of standard treatment modalities in older patients $(9,10)$. Due to the heterogeneity in the health background of elderly patients, treatment should be based on the level of fitness, frailty, or vulnerability.

Therefore, in this study, we aimed to identify predictive factors significantly correlating with the OS of older patients with wild-type EGFR advanced NSCLC.

We present the following article/case in accordance with the STROBE reporting checklist (available at http://dx.doi. org/10.21037/tlcr-20-894).

\section{Methods}

\section{Study design and patients}

This multicenter, retrospective cohort study was conducted among elderly patients (aged $\geq 75$ years) diagnosed with advanced lung cancer at Kyoto Prefectural University Hospital, Otsu City Hospital, and Japanese Red Cross Kyoto Daiichi Hospital, Japan, between January 2013 and December 2018. The study was conducted in accordance with the Declaration of Helsinki (as revised in 2013). The study was approved by Independent Ethics Committees of Kyoto Prefectural University of Medicine (ERB-C-1154) and each hospital, and individual consent for this retrospective analysis was waived.

We included patients regardless of receipt of prior cytotoxic chemotherapy. There were 124 patients with histopathologically or cytologically diagnosed NSCLC who were wild-type EGFR. Patients could also be negative for other driver gene mutations. Enrolled patients voluntarily opted for chemotherapy or palliative care after discussion with their physicians. The following variables were included in the retrospective analysis: age, gender, Eastern Cooperative Oncology Group performance status (ECOG PS), tumor-node-metastasis (TNM) stages, and OS. TNM stage was classified using the TNM stage classification system version 7 . Patients undergoing chemotherapy were in the advanced stage of cancer, while those receiving palliative care could be of any stage.

Pulmonologists were responsible for diagnosis and treatment selection as well as subsequent follow-ups.

\section{Statistical analysis}

IBM SPSS Advanced Statistics software (version 25; SPSS Inc., Chicago, IL, USA) was used for all statistical analyses. Patients' characteristics at the time of diagnosis are presented as standard descriptive statistics. Observed frequencies in the categorical variables were calculated. OS time after diagnosis was analyzed using Kaplan-Meier estimates, and differences in survival distributions for different patient subgroups were tested using log-rank tests. OS was considered at the date of the last visit for patients who were still alive without any documented disease progression. Cox proportional hazards models including age, ECOG PS, TNM staging, serum albumin levels, chemotherapy received (yes/no) were created. P values of $>0.05$ were considered to indicate statistical significance.

\section{Results}

\section{Baseline patient characteristics}

Of the 124 patients with NSCLC, 75 received best supportive care (BSC) without any specific therapy for lung cancer. Forty-nine patients received mono-chemotherapy or platinum doublet chemotherapy, including immune checkpoint inhibitors (ICIs). The median patient age was 
Table 1 BSC patient characteristics without EGFR mutation $(\mathrm{n}=75)$

\begin{tabular}{|c|c|}
\hline Items & Data \\
\hline Age (years), median [range] & $83[75-94]$ \\
\hline \multicolumn{2}{|l|}{ Sex, n (\%) } \\
\hline Male & $49(65.3)$ \\
\hline Female & $26(34.7)$ \\
\hline \multicolumn{2}{|l|}{ Pathology, n (\%) } \\
\hline Adenocarcinoma & $26(34.7)$ \\
\hline Squamous cell carcinoma & $24(32.0)$ \\
\hline Others & $25(33.3)$ \\
\hline \multicolumn{2}{|l|}{ Stage, n (\%) } \\
\hline Stage I & $5(6.7)$ \\
\hline Stage II & $4(5.3)$ \\
\hline Stage IIIA/IIIB & $7(9.3) / 4(5.3)$ \\
\hline Stage IV & $53(70.7)$ \\
\hline \multicolumn{2}{|l|}{ ECOG PS, n (\%) } \\
\hline 0 & $5(6.7)$ \\
\hline 1 & $22(29.3)$ \\
\hline 2 & 25 (33.3) \\
\hline 3 & $19(25.3)$ \\
\hline 4 & $4(5.3)$ \\
\hline OS (weeks), median [range] & $21[1-224]$ \\
\hline \multicolumn{2}{|l|}{ Reason for BSC, n (\%) } \\
\hline Older age & $26(34.7)$ \\
\hline Dementia & $7(9.3)$ \\
\hline Failure of PS & $30(40.0)$ \\
\hline Patient's wish & $12(16.0)$ \\
\hline
\end{tabular}

BSC, best supportive care; ECOG PS, Eastern Cooperative Oncology Group performance status.

83 years (range, 75-94 years) and 78 years (range, 7585 years) in the BSC and chemotherapy groups, respectively.

In the BSC group, 49 (65\%) patients were male. The histological subtypes were adenocarcinoma in 26 patients (34\%), squamous cell carcinoma in 24 patients (32\%), and other carcinomas in 25 patients (33\%). Twenty-seven patients (36\%) had an ECOG PS of 0 or 1 . Reasons for receiving BSC were poor PS in 30 patients (40\%), advanced age in 26 patients (34\%), dementia in seven patients $(9.3 \%)$,
Table 2 Chemotherapy patient characteristics without EGFR mutation $(\mathrm{n}=49)$

\begin{tabular}{lc}
\hline Items & Data \\
\hline Age (years), median [range] & 78 [75-85] \\
Sex, n (\%) & $37(75.5)$ \\
Male & $12(24.5)$ \\
Female & \\
Pathology, n (\%) & $28(57.1)$ \\
Adenocarcinoma & $12(24.4)$ \\
Squamous cell carcinoma & $9(18.4)$ \\
Others & \\
Stage, n (\%) & $16(32.6)$ \\
Stage III & $33(67.3)$ \\
Stage IV & \\
ECOG PS, n (\%) & $13(26.5)$ \\
0 & $29(59.2)$ \\
1 & $6(12.2)$ \\
2 & $1(1.3)$ \\
3 & $46-229]$ \\
\hline ECOG PS, Eastern Cooperative Oncology Group performance \\
status.
\end{tabular}

and patients' wish in 12 patients (16\%). Fifty-three patients (71\%) had stage IV disease, and only nine patients (12\%) were in the early stage (stage I or II). The median OS in the BSC group was 21 weeks (1-224 weeks).

In the chemotherapy group, 37 (75\%) patients were male. The histological subtypes were adenocarcinoma in 28 patients (57\%), squamous cell carcinoma in 12 patients (24\%), and other types of carcinomas in nine patients. Forty-two patients (86\%) had an ECOG PS of 0 or 1 . The median OS in all chemotherapy patients was 41 weeks (6-229 weeks). Of 49 patients that received chemotherapy, $21(42 \%)$ received platinum doublets, 25 (51\%) received third-generation cytotoxics (e.g., pemetrexed, docetaxel, paclitaxel, and nab-paclitaxel), and only three patients received ICI monotherapy.

The baseline clinicopathological characteristics of the patients in both groups are summarized in Tables 1 and 2, respectively. 

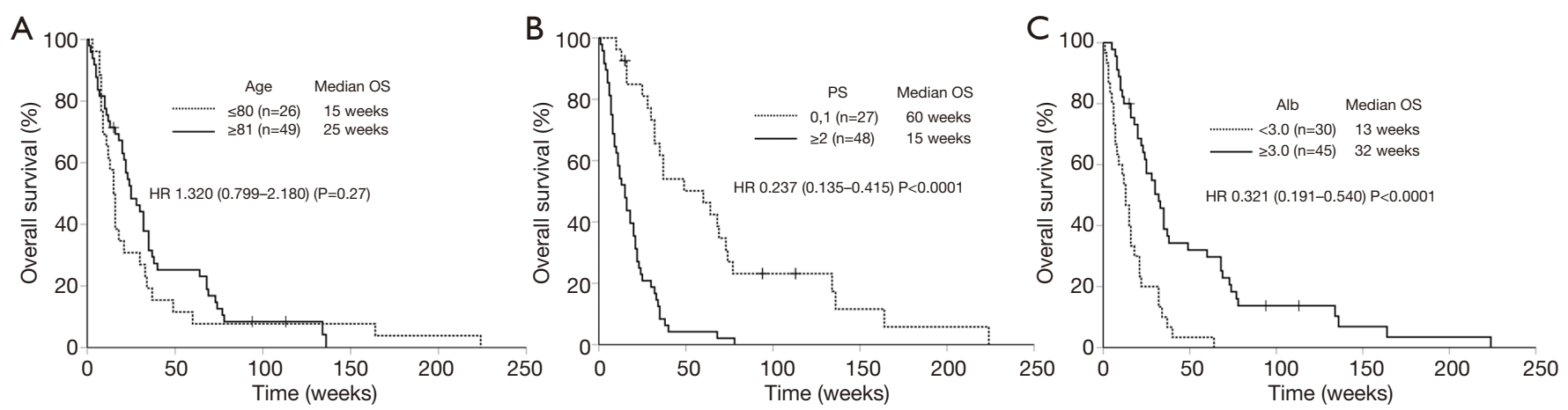

Figure 1 Overall survival of BSC patients according to (A) age, (B) PS and (C) albumin. (A) Age at the time of diagnosis is not associated with survival in patients receiving BSC. (B,C) OS of patients with good PS (PS=0.1) and high Alb level (Alb $\geq 3.0)$ show long survival period. BSC, best supportive care; PS, performance status.

Table 3 Cox proportional hazards models (BSC group, $\mathrm{N}=75$ )

\begin{tabular}{lcc}
\hline & & OS (weeks) \\
\cline { 2 - 3 } Items & $\mathrm{HR}(95 \% \mathrm{Cl})$ & $\mathrm{P}$ value \\
\hline ECOG PS (PS 3, 4 vs. PS 0, 1, 2) & $0.346(0.193-0.620)$ & $<0.001$ \\
Alb $(\geq 3.0$ vs. $<3.0 \mathrm{~g} / \mathrm{dL})$ & $2.543(1.469-4.400)$ & 0.001 \\
Over Stage IIIB vs. others & $0.333(0.162-0.687)$ & 0.003 \\
Age $(\geq 81$ vs. $<81 \mathrm{yr})$ & $1.110(0.66-1.908)$ & 0.705 \\
\hline
\end{tabular}

BSC, best supportive care; ECOG PS, Eastern Cooperative Oncology Group performance status.

\section{Predictive factors for $O S$ in patients receiving BSC}

To evaluate whether the clinical features are predictive of OS in the BSC group, we investigated the association between clinical parameters of interest and OS. We first examined the association between age and OS in patients receiving BSC (Figure 1). In the BSC group, 26 patients $(35 \%)$ were aged 80 years or less, and 49 patients $(65 \%)$ were aged 81 years and above. There was no significant difference in median OS between the group aged 80 years or below and the group aged 81 years and above; the median OS in the two groups was 25 and 15 weeks, respectively (hazard ratio: $1.32,95 \%$ confidence interval: $0.799-2.18, \mathrm{P}=0.27$; Figure $1 A$ ). This shows that age at the time of diagnosis is not associated with survival in patients receiving BSC. Patients with PS of 0 or 1 at the time of diagnosis $(n=27)$ had prolonged OS, compared to those with PS of 2 and above $(n=48)$, i.e., median of 60 weeks and 15 weeks, respectively (hazard ratio: $0.237,95 \%$ confidence interval: $0.135-0.415, \mathrm{P}<0.0001$; Figure $1 B$ ).

OS was longer in patients with albumin level $\geq 3.0 \mathrm{~g} / \mathrm{dL}$ at the time of diagnosis $(n=45)$ than in those with albumin level $<3.0 \mathrm{~g} / \mathrm{dL}(\mathrm{n}=30)$, i.e., median of 32 weeks and 13 weeks, respectively (hazard ratio: $0.321,95 \%$ confidence interval: 0.191-0.540, $\mathrm{P}<0.0001$; Figure 1C).

In the analysis of Cox proportional hazards models, median OS in patients receiving BSC was significantly correlated with PS $(\mathrm{P}<0.001)$ and albumin $(\geq 3.0 \mathrm{~g} / \mathrm{dL}$, $\mathrm{P}<0.001)$. There was no significant relationship between the age at the time of diagnosis and OS in the BSC group (age $<81$ years, $\mathrm{P}=0.705$, Table 3).

\section{Does chemotherapy significantly prolong OS in elderly patients?}

To evaluate the efficacy of chemotherapy in older patients with lung cancer, we compared the OS of the BSC and chemotherapy groups. To match the background of both groups, we selected cases with PS $=0-2$ and those with stage III or higher in the BSC group (Figure 2). Patients in the chemotherapy group $(\mathrm{n}=48)$ showed prolonged OS, compared to those in the BSC group ( $n=42)$; median OS was 48 weeks and 25 weeks, respectively (hazard ratio: 0.508 , 

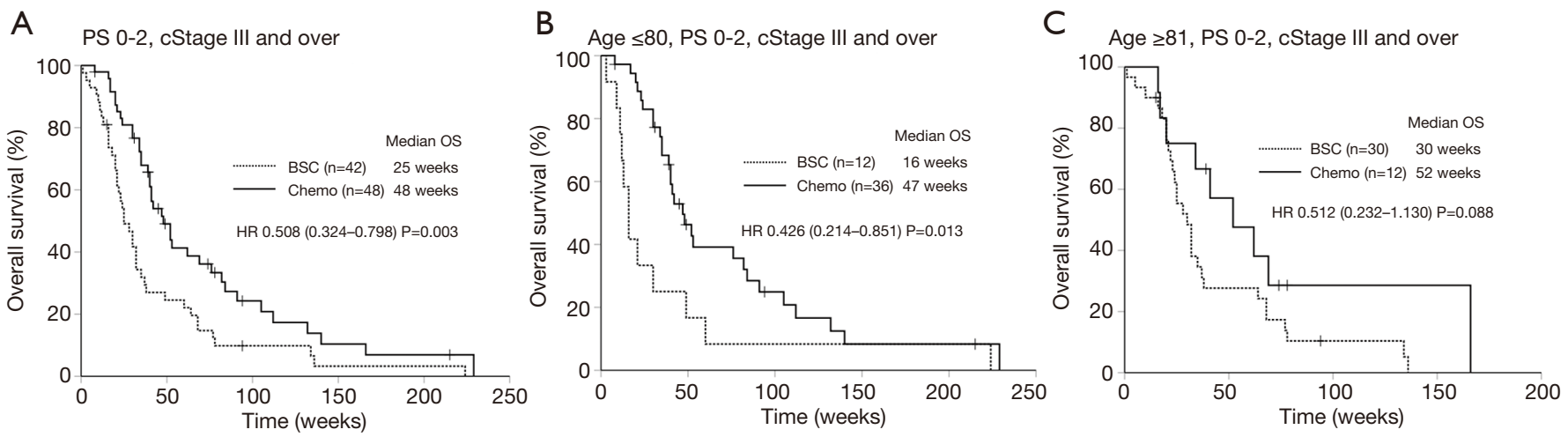

Figure 2 OS according to BSC or chemotherapy with the same background (PS =0, 1, 2 and case of stage III and over). In particular, (B) with 80 years and under, $(\mathrm{C})$ with 81 and over. $(\mathrm{A}, \mathrm{B})$ Patients in chemotherapy group had prolonged OS, compared to those in the BSC group. (C) Patients with 81 and over in chemotherapy group show tended to have longer OS than did the BSC group, but there was no significant difference between the two groups. OS, overall survival; BSC, best supportive care; PS, performance status.

Table 4 Cox proportional hazards models (over 81 years old, $\mathrm{N}=61$ )

\begin{tabular}{lcc}
\hline & & OS (weeks) \\
\cline { 2 - 3 } Items & HR (95\% Cl) & P value \\
\hline ECOG PS (PS 3, 4 vs. PS 0, 1, 2) & $0.291(0.156-0.543)$ & $<0.001$ \\
Alb $(\geq 3.0$ vs. $<3.0 \mathrm{~g} / \mathrm{dL})$ & $3.729(1.960-7.094)$ & $<0.001$ \\
Over Stage IIIB vs. others & $0.397(0.182-0.868)$ & 0.021 \\
Chemotherapy vs. BSC & $2.198(0.980-4.930)$ & 0.056 \\
\hline
\end{tabular}

ECOG PS, Eastern Cooperative Oncology Group performance status; BSC, best supportive care.

95\% confidence interval: $0.324-0.798, \mathrm{P}=0.003$; Figure $2 A$ ). The same relationship was also shown in those aged 80 years and below (hazard ratio: $0.426,95 \%$ confidence interval: $0.214-0.851, \mathrm{P}=0.013$; Figure $2 B$ ). Even in patients aged 81 years and above, the chemotherapy group tended to have longer OS than did the BSC group, but there was no significant difference in median OS between the two groups due to the very small number of subjects; $n=30$ and 12, the median OS was 52 weeks and 30 weeks, respectively (hazard ratio: $0.512,95 \%$ confidence interval: $0.232-1.13$, $\mathrm{P}=0.088$; Figure $2 C$ ). According to Cox proportional hazards analysis in older patients aged 81 years and above, OS was significantly associated with low PS $(\mathrm{P}<0.001)$ and high albumin levels $(\geq 3.0 \mathrm{~g} / \mathrm{dL}, \mathrm{P}<0.001)$, but not with chemotherapy $(\mathrm{P}=0.056$, Table 4).

\section{Discussion}

From January 2013 to October 2018, in our hospital, 37\% of the hospitalized patients with lung cancer were aged $>75$ years, and approximately $20 \%$ were aged $>80$ years (Figure 3). In our study, a relationship between age at the time of diagnosis and OS was not observed in the BSC group. In addition, PS and albumin levels at the time of diagnosis of lung cancer were the strongest predictive factors of OS in the BSC group.

Older patients should not be denied access to effective therapies due to age alone. Previous studies report that older people with good PS can be treated with chemotherapy to the same extent as that of younger people and can have a similar prognosis; thus, the prognosis with chemotherapy is better than that with BSC $(11,12)$. If the administration of BSC rather than surgery, radiation therapy, or chemotherapy is decided after the diagnosis of lung cancer, clinicians should carefully consider whether the treatment is adequate with reference to the guidelines. There are no clear criteria to opt for BSC over active treatment.

A recent study has shown that among relapsed 


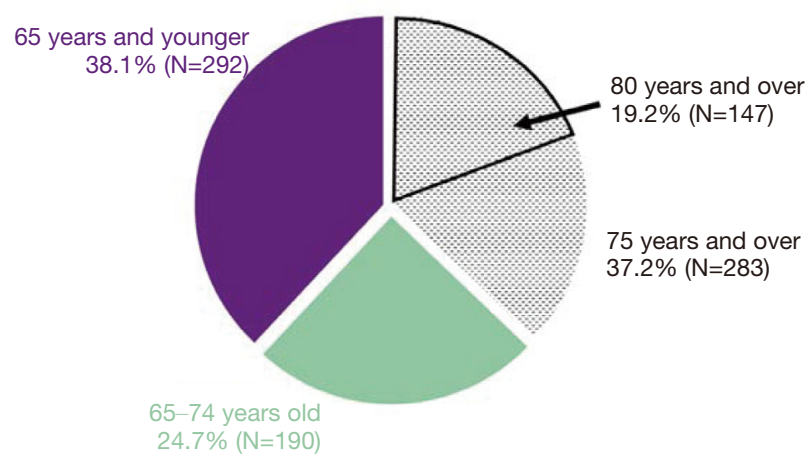

Figure 3 Hospitalized lung cancer patient in Kyoto Prefectural University of Medicine (Jan. 2013-Oct. 2018). It shows that 37\% of the hospitalized patients with lung cancer were aged $>75$ years, and approximately $20 \%$ were aged $>80$ years.

older patients with SCLC who received second-line chemotherapy, a substantially long OS may be expected in patients with good PS, with early stage disease at the time of starting first-line therapy, and with a longer interval between the start of first-line therapy to that of secondline chemotherapy (13). PS is the major prognostic factor in lung cancer, but it cannot accurately predict outcome in older patients who are treated with chemotherapy (14). Meanwhile, Blanc-Bisson et al. reported that malnutrition might increase mortality even in older patients with progression-free tumors (15). The patients with anemia, low albumin levels, and high NLR had shorter survival than did those without these factors (16).

In deciding between providing and not providing active treatment to older patients, it is important to assess whether they are more likely to die of lung cancer or conditions related to age. In our study, patients of the BSC group with low albumin levels had a shorter survival. In fact, patients with albumin $\geq 3.0$ and those with a good PS ( 0 or 1 ) may have a long survival period, even if they choose BSC for their lung cancer. The number of older patients with lung cancer will further increase in the future; hence, it is important to give enough information and comprehensively evaluate the patient's general condition and the patient's wish.

Our study demonstrated that the most common reason for choosing BSC was a decrease in PS ( $n=30,40 \%)$; this was followed by advanced age $(n=26,34.7 \%)$, desire of the patient $(\mathrm{n}=12,16 \%)$, and cognitive decline $(\mathrm{n}=7,9.3 \%)$. Older patients with lung cancer have a decline in systemic function with age and various comorbidities that can be fatal. As the current standard chemotherapy is based on the results of clinical studies mainly in patients aged 75 years and younger, there is a limitation in its use in older patients. Importantly, among geriatric medical services, older people mainly prioritize the maintenance of their quality of life, while they give the lowest priority to life extension and mortality reduction (17). Among patients with metastatic NSCLC, patients receiving early palliative care had less aggressive care at the end of life but longer survival than did patients receiving standard care (18). Therefore, multidisciplinary medical treatment from an early stage is desirable for older patients with cancer.

In the present study, older patients with advanced lung cancer treated with chemotherapy showed prolonged OS, compared to those receiving BSC. In patients aged 81 years and above, the chemotherapy group tended to have longer OS than did the BSC group, but there was no significant difference in the median OS between the two groups due to the small number of subjects. As older people advance in age, their individual diversity and individual differences increase; hence, treatment policies should be carefully decided for those aged 81 years and above.

Japanese guidelines define those aged 75 years and above as older people and recommend the administration of kinase inhibitors for gene mutation-positive lung cancer in such patients (19). Similarly, ICIs often have relatively mild adverse effects compared to conventional cytotoxic agents, and they are used in many older patients in clinical practice. In our study, three patients were able to complete treatment with ICIs without complications. If gene mutation is negative and programmed cell death ligand 1 is $<50 \%$ or unknown, it is strongly recommended that older people with good PS be treated with a single third-generation cytotoxic anticancer drug. This recommendation reflects the successful outcome of the third-generation cytotoxic anti-cancer drug docetaxel in clinical trials conducted in older Japanese patients (20). It is very difficult to predict the physical function in older people above 80 years of age. Older patients are often excluded from the clinical trials due to the perception that they are unable to tolerate aggressive chemotherapy and are more likely to suffer increased toxicity, with a resultant poor quality of life. In our study, the median OS of all older patients with lung cancer was significantly associated with PS and albumin level $\geq 3.0 \mathrm{~g} / \mathrm{dL}$ in the univariate analyses; however, chemotherapy intervention, age, and staging at the time of diagnosis were not associated with OS.

Treatment for cancer in older patients can be identical 
to that in the younger patients, and the same treatment outcome can be expected in the elderly in a good general condition $(10,21,22)$; however, complications related to the treatment might increase (23). There are only a limited number of studies on chemotherapy for older patients with lung cancer; thus, our study contributes towards increasing the data generated thus far and enhancing our understanding of treatment approaches for this difficult-totreat population.

Immunotherapies are also well tolerated, and further treatment options for advanced lung cancer are expected to increase for older patients as well. In this retrospective study, three immunotherapeutic agents were included.

If the patient is expected to benefit from chemotherapy, the patient's cognitive ability and physical function should be evaluated to check whether chemotherapy could be applied. Functional evaluation of older patients for cancer treatment is divided into three categories. First, older patients are considered fit if they can receive standard treatment similar to that in young adults. Second, older patients are deemed unfit when the standard cancer treatment cannot be provided (frail patients). The third category comprises patients in whom cancer treatment can be administered to some extent (vulnerable patients) (24). Comprehensive geriatric assessment (CGA) consists of activities of daily living (meal, changing clothes, etc.), instrumental activities of daily living (shopping, talking on the telephone etc.), cognitive ability, happiness, communication, and social environment. In a randomized controlled study that examined the usefulness of CGA to determine the line of treatment in NSCLC in those aged 70 years and above, there was no significant difference in OS between chemotherapy allocation on the basis of PS and age (standard arm) and treatment allocation on the basis of CGA (CGA arm) (25). However, patients in the CGA compared to those in the standard arm, experienced significantly less all grade toxicity and had fewer occurrences of treatment failure due to toxicity. Furthermore, in the study by Kalsi et al., CGA interventions were associated with improved chemotherapy tolerance (26). Appropriate intervention for adverse events after CGA might improve the outcomes in older patients.

Our study had several limitations. First, there was a potential bias related to the multicenter design; however, all the facilities are core hospitals with no difference. Second, our study involved a small number of subjects analyzed in a retrospective manner. Therefore, studies involving more cases are necessary in the future. Third, the effects of other medical comorbidities that may affect OS (renal dysfunction, diabetes mellitus, etc.) were not included in the analysis and could affect the validity of our findings.

\section{Conclusions}

In this study, we retrospectively evaluated the treatment for advanced NSCLC with EGFR and/or other mutations negative in older patients. There was no relationship between age at the time of diagnosis and OS in the BSC group. In addition, PS and albumin levels at the time of diagnosis of lung cancer were the most prognostic factors for OS in the BSC group. In our study, patients with a good performance status and high albumin levels had a long survival period even if they chose BSC to manage lung cancer. Patients aged 81 years and above tended to have a longer OS in the chemotherapy group than in the BSC group, but the number of subjects was small, and the difference was not statistically significant. As older people advance in age, their individual diversity and individual differences increase; hence, treatment policies should be carefully decided for those aged 81 years and above. The number of older patients with lung cancer will increase further in the future, so it is important to give enough information and to comprehensively evaluate the patient's general condition and the patient's wish.

\section{Acknowledgments}

The authors sincerely appreciate all the physicians and patients who participated in this study.

Funding: None.

\section{Footnote}

Reporting Checklist: The authors have completed the STROBE reporting checklist. Available at http://dx.doi. org/10.21037/tlcr-20-894

Data Sharing Statement: Available at http://dx.doi. org/10.21037/tlcr-20-894

Peer Review File: Available at http://dx.doi.org/10.21037/ tlcr-20-894

Conflicts of Interest: All authors have completed the ICMJE uniform disclosure form (available at http://dx.doi. org/10.21037/tlcr-20-894). Dr. TY reports receiving 
research grants from Ono Pharmaceutical Co., Ltd., Takeda Pharmaceutical Co. Ltd., Pfizer Inc., Boehringer Ingelheim Japan Inc., and Chugai Pharmaceutical Co., Ltd. Dr. JU reports receiving research grants from Eli Lilly Japan K.K., AstraZeneca K.K., and Boehringer Ingelheim Japan Inc. Dr. KT reports receiving research grants from ChugaiRoche Co., and Ono Pharmaceutical Co., and personal fees from AstraZeneca Co., Chugai-Roche Co., MSD-Merck Co., Eli Lilly Co., Boehringer-Ingelheim Co., and DaiichiSankyo Co. The other authors have no conflicts of interest to declare.

Ethical Statement: The authors are accountable for all aspects of the work in ensuring that questions related to the accuracy or integrity of any part of the work are appropriately investigated and resolved. The study was conducted in accordance with the Declaration of Helsinki (as revised in 2013). The study was approved by Independent Ethics Committees of Kyoto Prefectural University of Medicine (ERB-C-1154) and each hospital, and individual consent for this retrospective analysis was waived.

Open Access Statement: This is an Open Access article distributed in accordance with the Creative Commons Attribution-NonCommercial-NoDerivs 4.0 International License (CC BY-NC-ND 4.0), which permits the noncommercial replication and distribution of the article with the strict proviso that no changes or edits are made and the original work is properly cited (including links to both the formal publication through the relevant DOI and the license). See: https://creativecommons.org/licenses/by-nc-nd/4.0/.

\section{References}

1. WHO. The top 10 causes of death [Internet]. [cited 2020 Jul 14]. Available online: https://www.who.int/news-room/ fact-sheets/detail/the-top-10-causes-of-death

2. Owonikoko TK, Ragin CC, Belani CP, et al. Lung cancer in elderly patients: An analysis of the surveillance, epidemiology, and end results database. J Clin Oncol 2007;25:5570-7.

3. Hori M, Matsuda T, Shibata A, et al. Cancer incidence and incidence rates in Japan in 2009: a study of 32 populationbased cancer registries for the Monitoring of Cancer Incidence in Japan (MCIJ) project. Jpn J Clin Oncol 2015;45:884-91.

4. Maemondo M, Minegishi Y, Inoue A, et al. First-line gefitinib in patients aged 75 or older with advanced non- small cell lung cancer harboring epidermal growth factor receptor mutations: NEJ 003 study. J Thorac Oncol 2012;7:1417-22.

5. Gridelli C, Perrone F, Gallo C, et al. Chemotherapy for elderly patients with advanced non-small-cell lung cancer: The multicenter Italian lung cancer in the elderly study (MILES) phase III randomized trial. J Natl Cancer Inst 2003;95:362-72.

6. Kudoh S, Takeda K, Nakagawa K, et al. Phase III study of docetaxel compared with vinorelbine in elderly patients with advanced non-small-cell lung cancer: Results of the West Japan Thoracic Oncology Group trial (WJTOG 9904). J Clin Oncol 2006;24:3657-63.

7. Sacher AG, Le LW, Leighl NB, et al. Elderly patients with advanced NSCLC in phase III clinical trials: Are the elderly excluded from practice-changing trials in advanced NSCLC? J Thorac Oncol 2013;8:366-8.

8. Jørgensen TL, Hallas J, Land LH, et al. Comorbidity and polypharmacy in elderly cancer patients: The significance on treatment outcome and tolerance. J Geriatr Oncol 2010;1:87-102.

9. Abe T, Takeda K, Ohe Y, et al. Randomized phase III trial comparing weekly docetaxel plus cisplatin versus docetaxel monotherapy every 3 weeks in elderly patients with advanced non-small-cell lung cancer: the intergroup trial JCOG0803/WJOG4307L. J Clin Oncol 2015;33:575-81.

10. Quoix E, Zalcman G, Oster JP, et al. Carboplatin and weekly paclitaxel doublet chemotherapy compared with monotherapy in elderly patients with advanced non-smallcell lung cancer: IFCT-0501 randomised, phase 3 trial. Lancet 2011;378:1079-88.

11. Earle CC, Tsai JS, Gelber RD, et al. Effectiveness of chemotherapy for advanced lung cancer in the elderly: instrumental variable and propensity analysis. J Clin Oncol 2001;19:1064-70.

12. Non-small Cell Lung Cancer Collaborative Group. Chemotherapy in non-small celi lung cancer: a metaanalysis usmg updated data on individual patients from 52 randomised clinical trials Introduction Worldwide more than half a million new cases of. BMJ 1995;311:899-909.

13. Igawa S, Naoki K, Shintani Y, et al. Survival and prognostic factors in elderly patients receiving second-line chemotherapy for relapsed small-cell lung cancer: Results from the Japanese Joint Committee of Lung Cancer Registry. Lung Cancer 2020;146:160-4.

14. Maione P, Perrone F, Gallo C, et al. Pretreatment quality of life and functional status assessment significantly predict survival of elderly patients with advanced non-small-cell 
lung cancer receiving chemotherapy: A Prognostic analysis of the multicenter italian lung cancer in the elderly study. J Clin Oncol 2005;23:6865-72.

15. Blanc-Bisson C, Fonck M, Rainfray M, et al. Undernutrition in elderly patients with cancer: Target for diagnosis and intervention. Crit Rev Oncol Hematol 2008;67:243-54.

16. Kaya V, Yildirim M, Demirpence O, et al. Prognostic significance of basic laboratory methods in non- smallcell-lung cancer. Asian Pac J Cancer Prev 2013;14:5473-6.

17. Roberts H, Khee TS, Philp I. Setting priorities for measures of performance for geriatric medical services. Age Ageing 1994;23:154-7.

18. Gaertner J, Wolf J, Voltz R. Early palliative care for patients with metastatic cancer. Curr Opin Oncol 2012;24:357-62.

19. Akamatsu H, Ninomiya K, Kenmotsu H, et al. The Japanese Lung Cancer Society Guideline for non-small cell lung cancer, stage IV. Int J Clin Oncol 2019;24:731-70.

20. Barnett K, Mercer SW, Norbury M, et al. Epidemiology of multimorbidity and implications for health care, research, and medical education: A cross-sectional study. Lancet 2012;380:37-43.

21. Folprecht G, Seymour MT, Saltz L, et al. Irinotecan/ fluorouracil combination in first-line therapy of older

Cite this article as: Seto Y, Kaneko Y, Mouri T, Fujii H, Tanaka S, Shiotsu S, Hiranuma O, Morimoto Y, Iwasaku M, Yamada T, Uchino J, Takayama K. Prognostic factors in older patients with wild-type epidermal growth factor receptor advanced non-small cell lung cancer: a multicenter retrospective study. Transl Lung Cancer Res 2021;10(1):193-201. doi: 10.21037/ tlcr-20-894 and younger patients with metastatic colorectal cancer: Combined analysis of 2,691 patients in randomized controlled trials. J Clin Oncol 2008;26:1443-1451.

22. Goldberg RM, Tabah-Fisch I, Bleiberg H, et al. Pooled analysis of safety and efficacy of oxaliplatin plus fluorouracil/leucovorin administered bimonthly in elderly patients with colorectal cancer. J Clin Oncol 2006;24:4085-91.

23. Gajra A, Klepin HD, Feng T, et al. Predictors of chemotherapy dose reduction at first cycle in patients age 65 years and older with solid tumors. J Geriatr Oncol 2015;6:133-40.

24. Mizutani T, Nakamura K, Fukuda H, et al; Geriatric Study Committee/Japan Clinical Oncology Group. Geriatric Research Policy: Japan Clinical Oncology Group (JCOG) policy. Jpn J Clin Oncol 2019;49:901-10.

25. Corre R, Greillier L, Le Caër H, et al. Use of a comprehensive geriatric assessment for the management of elderly patients with advanced non-small cell lung cancer: The Phase III randomized ESOGIA-GFPC-GECP 08-02 Study. J Clin Oncol 2016;34:1476-83.

26. Kalsi T, Babic-Illman G, Ross PJ, et al. The impact of comprehensive geriatric assessment interventions on tolerance to chemotherapy in older people. Br J Cancer 2015;112:1435-44. 